\title{
Role of $\mathrm{Fe}_{2} \mathrm{~B}$ phase on the magnetomechanical behavior of Fe based materials
}

\author{
Cristina BORMIO-NUNES*, Olivier HUBERT ${ }^{\dagger}$ \\ *Escola de Engenharia de Lorena, Universidade de São Paulo Estrada Municipal do Campinho s/n 12602-810 Lorena, Brazil \\ ${ }^{\dagger}$ LMT-Cachan (ENS Cachan/CNRS/Université Paris-Saclay) 61 avenue du Président Wilson F-94235 Cachan, France \\ Email: cristina@demar.eel.usp.br, hubert@1mt.ens-cachan.fr
}

\begin{abstract}
This paper reports magnetic, magnetostrictive and piezomagnetic experimental results performed on a pure iron and $\mathrm{Fe}-\mathrm{B}$ alloy, and associated modeling. Results allow a better understanding of the role of $\mathrm{Fe}_{2} \mathrm{~B}$ phase in $\mathrm{Fe}-\mathrm{Al}-\mathrm{B}$ alloys.
\end{abstract}

\section{INTRODUCTION}

Magnetic sensors and actuators are usually made of materials that present large coupling between mechanical and magnetic properties (i.e. magnetostriction and/or piezomagnetic behavior). Rare earth elements ( $\mathrm{Tb}, \mathrm{Dy})$ are often used as secondary elements in alloys that are employed, because they exceptionally enhance the magnetomechanical properties of these materials. However, due to the increase of price and limited availability of these rare earth elements, the development of rare earth free alloys is relevant. Fe-Al alloys demonstrated to be interesting candidates [1] ${ }^{1}$. Recently an important increase of the $\mathrm{Fe}-\mathrm{Al}$ alloys magnetostriction has been observed due to the addition of boron [3]. The boron added to $\mathrm{Fe}-\mathrm{Al}$ alloys is not soluble in the cubic lattices, but causes the formation of the $\mathrm{Fe}_{2} \mathrm{~B}$ phase. The influence of boron content is however not fully understood and the analysis of this influence is complex due to the possible presence of a $\mathrm{Fe}_{3} \mathrm{Al}$ ordered phase for aluminum contents higher than $20 \mathrm{at} \%$. In the present work, the magnetic, magnetostrictive and piezomagnetic ${ }^{2}$ behavior of pure iron and pure iron containing $1.6 a t \%$ of boron are compared. Then a biphasic model is used to model the behaviors, associated to proper localization rules to define the local magnetic field and stress. The role of $\mathrm{Fe}_{2} \mathrm{~B}$ phase inside the Fe matrix is finally clarified.

\section{MATERIAL PRESENTATION AND EXPERIMENTAL DEVICES}

Pure iron sample is a commercial ARMCO iron (purity higher than $99.85 \%$ ) machined by spark erosion to form bars of dimensions: $120 \times 15 \times 2.5\left(\mathrm{~mm}^{3}\right)$. Fe-1.6at\%B alloy was produced by arc melting in argon atmosphere and re-melted in a high vacuum furnace inside a ceramic tube. The bar obtained had around $110 \mathrm{~mm}$ of length. Plates of thickness of $3 \mathrm{~mm}$ and $12 \mathrm{~mm}$ width were cut from the center in the longitudinal direction of the bars by spark erosion. The

\footnotetext{
${ }^{1}$ These alloys exhibit on the other hand high mechanical properties and good corrosion resistance [2].

${ }^{2}$ Piezomagnetic behavior is defined as variation of magnetization with stress at constant magnetic field [4], [5].
}

plates were annealed in inert atmosphere at $1100^{\circ} \mathrm{C}$ during 24 $\mathrm{h}$ and quenched in water. The microstructure of the samples were characterized by scanning electron microscopy: imaging by secondary electrons (SEM) and crystallographic texture by electron back-scattered diffraction (EBSD). The samples preparation for SEM observation consists of mechanical and subsequent electro polishing. Pure iron sample exhibits a homogeneous microstructure of equiaxed $\alpha$-phase grains of about $100 \mu \mathrm{m}$ mean diameter. EBSD measurements did not reveal any preferential orientation for this sample. The microstructure of $\mathrm{Fe}-1.6 \mathrm{at} \% \mathrm{~B}$ is presented in details in a recent paper [4]. Despite the final heat treatment the microstructure of the $\alpha$-phase is dendritic and presents large grains $(>400$ $\mu \mathrm{m})$. Microstructure observations and analyses confirm that all boron is concentrated in $\mathrm{Fe}_{2} \mathrm{~B}$ lamellas localized in the interdendritic micro-constituents.

The volume fraction of the phase $\mathrm{Fe}_{2} \mathrm{~B}$ was evaluated by image analyses and EBSD leading to a value close to $13 \%$. The EBSD measurements were made in each side of the plate in areas about $1.2 \mathrm{~mm}^{2}$, at the places where the strain gauges used for the magnetostriction measurement were glued. The material should exhibit some barely isotropic behavior since the average orientation of grains is close to $\langle 431>$.

The anhysteretic piezomagnetic behavior measurement set up acquires the induction (B) (or magnetization - M) and longitudinal magnetostriction $(\lambda)$ under different levels of applied stress varying the magnetic field. For each applied magnetic field, the sample is demagnetized [5]. The active ranges of stresses and magnetic field are $-50 \leq \sigma \leq 50 \mathrm{MPa}$ and $0<\mathrm{H}$ $<10 \mathrm{kA} / \mathrm{m}$, respectively. The system consists in a sample plate positioned inside a primary cylindrical coil. Two soft ferrite Uyokes close the magnetic circuit and one strain gauge is glued in each side of the plate to acquire the magnetostriction using a Wheatstone bridge [6]. The magnetization is measured thanks to a pick up coil wound in the central region of the plates close to the position of the strain gages. Hydraulic jaws of the tensile-compressive machine were used to grip the sample, to apply the stress.

\section{EXPERIMENTAL RESULTS}

Figure 1 shows the measured $B$ vs. $H$ curves for fixed values of stress $\sigma$ for both samples. We observe that the magnetization of the $\mathrm{Fe}-1.6 \mathrm{at} \% \mathrm{~B}$ sample is smaller than pure 


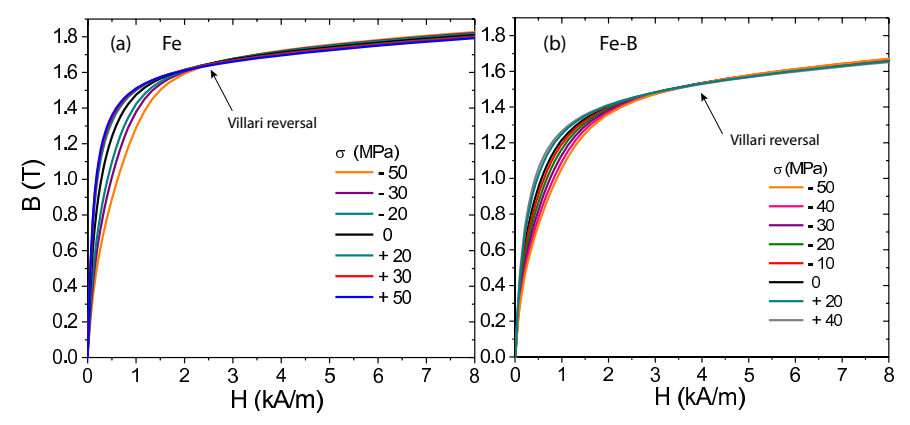

Fig. 1. Anhysteretic magnetic curves under applied stress of: (a) pure iron; (b) Fe-1.6at $\% \mathrm{~B}$ alloy.

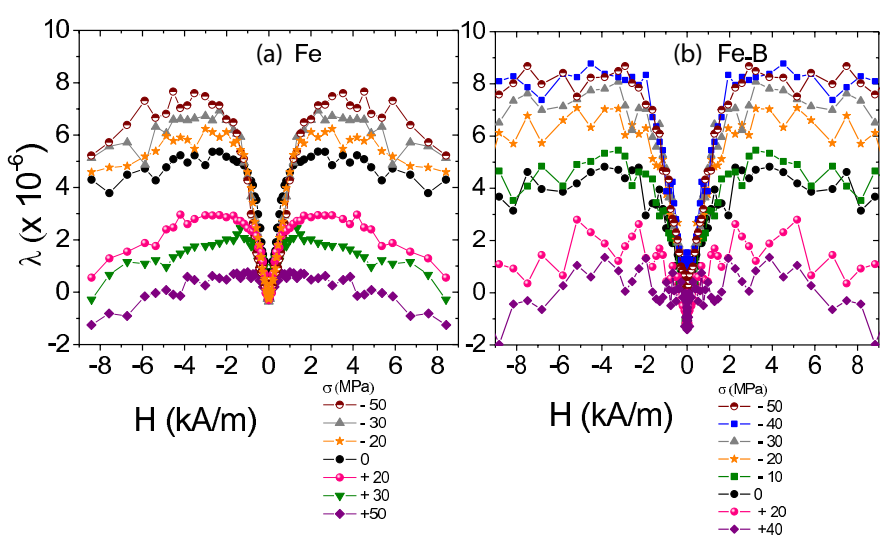

Fig. 2. Anhysteretic magnetostriction curves under applied stress of: (a) pure iron; (b) Fe-1.6at\%B alloy.

iron sample. The influence of stress on both samples seems very close: at low field, the variation of induction with respect to stress is positive, denoting a positive magnetostriction behavior; at higher field, the variation of induction with respect to stress becomes negative, associated to the so-called Villari reversal. It can be observed that the Villari reversal point is reached at a magnetic field level 2 times higher for Fe-1.6at\%B alloy than for pure iron. Transition seems on the other hand more field distributed. Figure 2 shows the measured $\lambda v s$. $H$ curves for fixed values of stress $\sigma$ for both samples. The magnetostrictive behavior is in accordance with the magnetic behavior considering that the Villari reversal point is reached for $d B / d \sigma=d \lambda / d H=0$ according to the thermodynamic equilibrium [7]. It can be noticed that results for pure iron meet the former results widespread in the literature (see for instance [8]). The introduction of boron has two major effects: i) it decreases apparently the average saturation magnetization; ii) it increases apparently the average saturation magnetostriction, leading to a global higher value of magnetostriction whatever the stress level and leading to the magnetic field shift of the Villari reversal point.

The $M v s$. $\sigma$ curves at constant $H$ for both alloys were built from the data of figure 1 for eight fixed values of magnetic field in the range of $0.5-8 \mathrm{kA} . \mathrm{m}^{-1}$ and are depicted in figure 3. Subsequently, from these $M$ vs. $\sigma$ curves the respective sensitivity $d M /\left.d \sigma\right|_{H}$ is calculated and figure 4 displays the

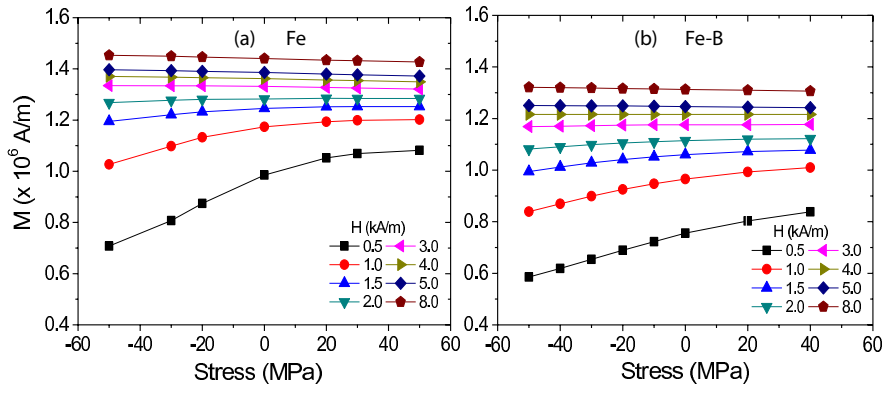

Fig. 3. Piezomagnetic curves at different magnetic field level of: (a) pure iron; (b)Fe-1.6at\%B alloy.
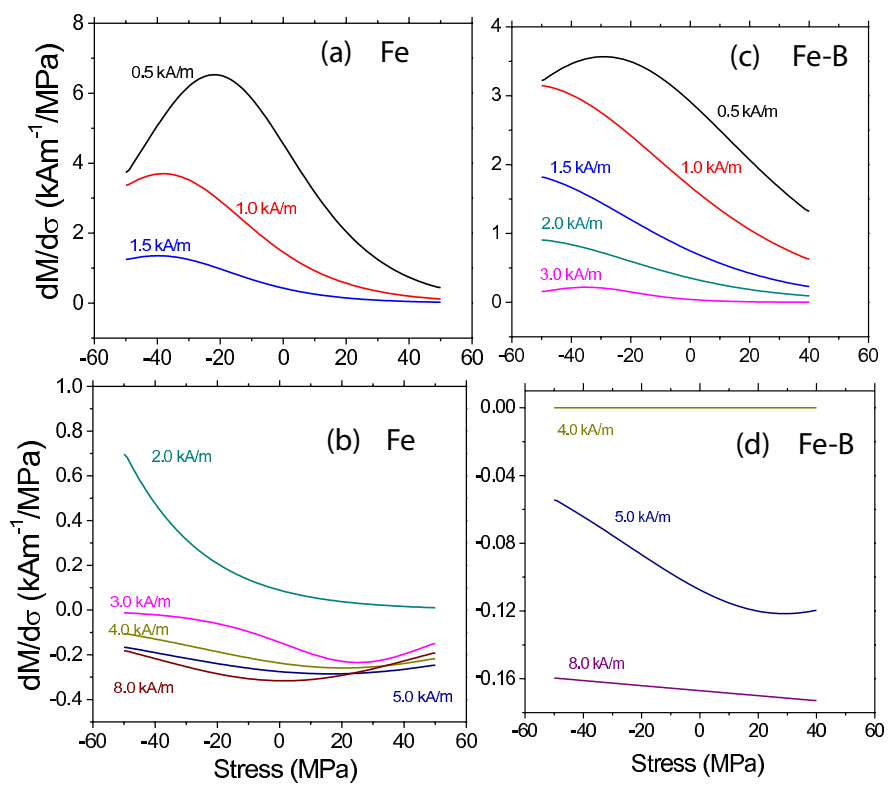

Fig. 4. Piezomagnetic sensitivity curves at different magnetic field level of: (a-b) pure iron; (c-d) $\mathrm{Fe}-1.6 \mathrm{at} \% \mathrm{~B}$ alloy.

associated plots at the same values of fixed applied field. The highest sensitivities are obtained at low magnetic field for pure iron sample (reaching $6 \mathrm{kA} . \mathrm{m}^{-1} / \mathrm{MPa}$ ). The magnetic field and stress ranges where this sensitivity is high is nevertheless wider for the Fe-B alloy. The piezomagnetic sensitvity of Fe$\mathrm{B}$ alloy is consequently globally enhanced comparing to pure iron sample.

\section{INFLUENCE OF THE $\mathrm{FE}_{2} \mathrm{~B}$ PHASE}

The $\mathrm{Fe}_{2} \mathrm{~B}$ phase is a tetragonal ferromagnetic phase exhibiting a high uniaxial anisotropy $\left(\mathrm{K}_{1}=-4.27 \times 10^{5}{\mathrm{~J} . \mathrm{m}^{-3}}^{-3}\right)$ at room temperature [9]. The saturation magnetization of $\mathrm{Fe}_{2} \mathrm{~B}$ phase is about $1.2 \times 10^{6} \mathrm{~A} / \mathrm{m}$ [10], lower than the saturation magnetization of iron, explaining the decrease of induction of $\mathrm{Fe}-1.6 \mathrm{at} \% \mathrm{~B}$ alloy comparing to pure iron. The saturation magnetostriction of $\mathrm{Fe}_{2} \mathrm{~B}$ phase is estimated to be $20 \mathrm{ppm}$ in polycrystalline samples and the magnetostriction is increasing monotonically with magnetic field until saturation [11]. The saturation magnetostriction of $\mathrm{Fe}_{2} \mathrm{~B}$ is much higher than the one of iron, explaining the enhancement of magnetostriction 
of $\mathrm{Fe}-1.6 \mathrm{at} \% \mathrm{~B}$ sample comparing to pure iron. The increase of magnetic field threshold where the Villari reversal occurs is an indirect consequence of the same phenomenon. However simple averaging rules do not allow to understand the enhancement of piezomagnetic sensitivity observed for $\mathrm{Fe}-1.6 \mathrm{at} \% \mathrm{~B}$ alloy comparing to pure iron. Some specific interaction must be considered between the $\mathrm{Fe}_{2} \mathrm{~B}$ phase and the matrix.

The presence of two different phases creates a local perturbation called demagnetizing field in magnetism and residual stress in mechanics. A medium composed of $i$ phases of volume fraction $f_{i}$ is considered. The local magnetic field applied to the phase $i$ is a complex function of macroscopic field $\vec{H}$ and the properties of the mean medium. In the case of spheroidal inclusion [12], the field is demonstrated as homogeneous on each phase. Considering on the other hand a linear susceptibility of average medium $\chi_{m}$, the local magnetic field in the phase $i$ is given by:

$$
\vec{H}_{i}=\vec{H}+\frac{1}{3+2 \chi_{m}}\left(\vec{M}-\vec{M}_{i}\right)=\vec{H}+\vec{H}_{i}^{d}
$$

where $\vec{M}$ is the average magnetization, $\vec{M}_{i}$ is the local magnetization. $\vec{H}_{i}^{d}$ is the so called demagnetizing field acting on phase $i$. The extension to nonlinear behavior involves to use the sequent susceptibility for the definition of $\chi_{m}$.

$$
\chi_{m}=\|\vec{M}\| /\|\vec{H}\|
$$

Averaging operations lead to

$$
\vec{H}=<f_{i} \vec{H}_{i}>\quad \text { and } \quad \vec{M}=<f_{i} \vec{M}_{i}>
$$

The stress localization formulation in case of a deformable matrix is due to Hill [13]. Equation 4 gives the stress field within the inclusion $i$ submitted to a macroscopic stress $\sigma . \varepsilon_{i}$ is the total strain tensor of the inclusion considered. $\varepsilon$ is the average total strain tensor over the volume.

$$
\sigma_{i}=\sigma+\mathbb{C}^{\star}\left(\varepsilon-\varepsilon_{i}\right)=\sigma+\sigma_{i}^{r}
$$

$\mathbb{C}^{\star}$ is the Hill's constraint tensor depending on the distribution and shape of inclusions and on the stiffness properties of materials. $\sigma_{i}^{r}$ is the so called residual stress tensor acting on inclusion $i$. If homogeneous isotropic elastic properties (Young's modulus $E$ and Poisson's ratio $v$ ), spherical shape inclusions and additivity of deformation (total deformation $=$ elastic deformation + magnetostrictive deformation) are considered, equation 4 can be simplified in:

$$
\sigma_{i}=\sigma+\frac{E(7-5 v)}{15\left(1-v^{2}\right)}\left(\varepsilon^{\mu}-\varepsilon_{i}^{\mu}\right)
$$

where $\varepsilon_{i}^{\mu}$ and $\varepsilon^{\mu}$ denote the local and average magnetostriction strain tensor respectively. Averaging operations lead to:

$$
\sigma=<f_{i} \sigma_{i}>\quad \text { and } \quad \varepsilon^{\mu}=<f_{i} \varepsilon_{i}^{\mu}>
$$

This approach is applied to the $\mathrm{Fe}-1.6 \mathrm{at} \% \mathrm{~B}$ sample with $f_{\alpha}$ and $f_{\mathrm{Fe}_{2} \mathrm{~B}}$ the volume fractions of $\alpha$ phase (pure iron) and $\mathrm{Fe}_{2} \mathrm{~B}$ phase respectively (with $f_{\mathrm{Fe}_{2} B}=13 \%$ ). The problem is next simplified in a $1 \mathrm{D}$ problem (all quantities measured along $x$ axis for example), the average magnetic and magnetization fields verify:

$$
H=f_{\alpha} H_{\alpha}+f_{F_{2} B} H_{F_{2} B}
$$

and

$$
M=f_{\alpha} M_{\alpha}+f_{\mathrm{Fe}_{2} B} M_{\mathrm{Fe}_{2} \mathrm{~B}}
$$

The average uniaxial stress and longitudinal magnetostriction strain $(\lambda)$ verify:

$$
\begin{aligned}
& \sigma=f_{\alpha} \sigma_{\alpha}+f_{F_{2} B} \sigma_{F_{2} B} \\
& \lambda=f_{\alpha} \lambda_{\alpha}+f_{F_{2} B} \lambda_{F_{2} B}
\end{aligned}
$$

The magnetic field inside the $\alpha$ phase is given by:

$$
H_{\alpha}=H+\frac{1}{3+2 \chi_{m}}\left(M-M_{\alpha}\right)
$$

An analysis detailed in [4] shows that the magnetization mechanisms of the $\mathrm{Fe}_{2} \mathrm{~B}$ phase begins by an easy magnetization rotation of magnetic moments inside the (001) planes, leading to a high initial susceptibility. The magnetic field level used in the experiments reported in the paper is however not enough to begin the macroscopic rotation. $\mathrm{Fe}_{2} \mathrm{~B}$ phase can consequently be considered as an isotropic very soft phase (negligible magneto-cystalline energy) with apparent saturation magnetization $\mathbf{M}_{s}^{F e_{2} B}=(\pi / 4) \mathbf{M}_{s}^{F e_{2} B}=9 \times 10^{-5} \mathrm{~A} \cdot \mathrm{m}^{-1}$ and apparent saturation magnetostriction $\lambda_{s}^{\prime} \mathrm{Fe}_{2} \mathrm{~B}=\lambda_{100}^{\prime \mathrm{Fe}_{2} \mathrm{~B}}=\lambda_{111}^{\prime} \mathrm{Fe}_{2} \mathrm{~B}=\frac{1}{2} \lambda_{s}^{\mathrm{Fe}_{2} \mathrm{~B}}$ $=10$ ppm. Because $\mathrm{Fe}_{2} \mathrm{~B}$ phase is very soft, $M_{\mathrm{Fe}_{2} \mathrm{~B}}>M$ so that, due to averaging, $M_{\alpha}<M$. The magnetic field in the $\alpha$ phase is consequently higher than the average magnetic field $\left(\mathrm{H}_{\alpha}>\mathrm{H}\right)$, enhancing both magnetization and magnetostriction. The stress field inside the $\alpha$ phase is:

$$
\sigma_{\alpha}=\sigma+\frac{E(7-5 v)}{15\left(1-v^{2}\right)}\left(\lambda-\lambda_{\alpha}\right)
$$

Due to soft magnetic properties, magnetostriction in the $\mathrm{Fe}_{2} \mathrm{~B}$ phase is higher than average magnetostriction at low magnetization level $\left(\lambda_{\mathrm{Fe}_{2} B}>\lambda\right)$, so that, due to averaging, $\lambda_{\alpha}<\lambda$. The stress field in the $\alpha$ phase is consequently higher than the average stress field $\left(\sigma_{\alpha}>\sigma\right)$. Considering an unloaded specimen $(\sigma=0)$, a positive stress is created inside the matrix counterbalanced by a negative stress field in the $\mathrm{Fe}_{2} \mathrm{~B}$ phase. The longitudinal magnetostriction being positive for $\alpha$-phase, the positive residual stress leads to enhanced magnetization and magnetostriction properties as well. This simplified composite approach allows to explain an enhancement effect on piezomagnetic properties. It is shown in [4] that the higher the magnetostriction of matrix, the stronger the enhancement. The low magnetostrictive properties of pure iron do not allow to clearly illustrate this effect in case of Fe-B alloy: the mixing rule gives similar results with or without localization.

\section{Modeling}

A two-scale reversible modeling of the magneto-mechanical behavior of each phase has been made, complemented by the localization and homogenization rules. Details of the model and physical quantities used are not presented here 

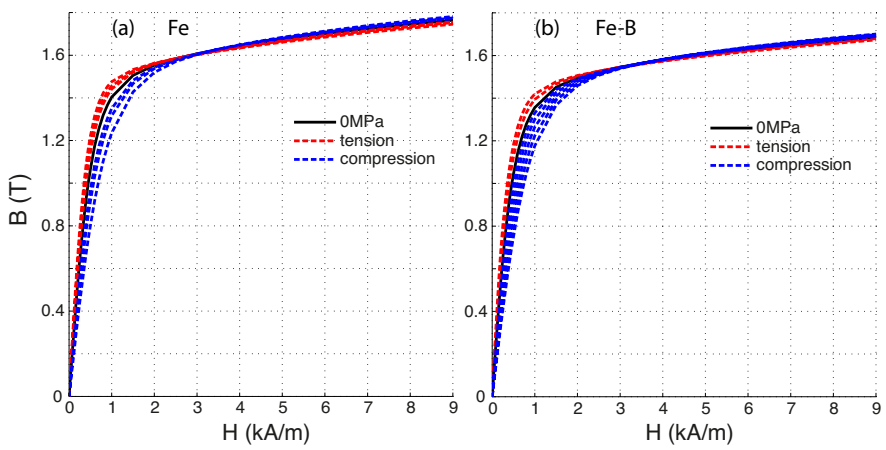

Fig. 5. Modeling - magnetic curves under applied stress of: (a) pure iron; (b)Fe-1.6at\%B alloy.

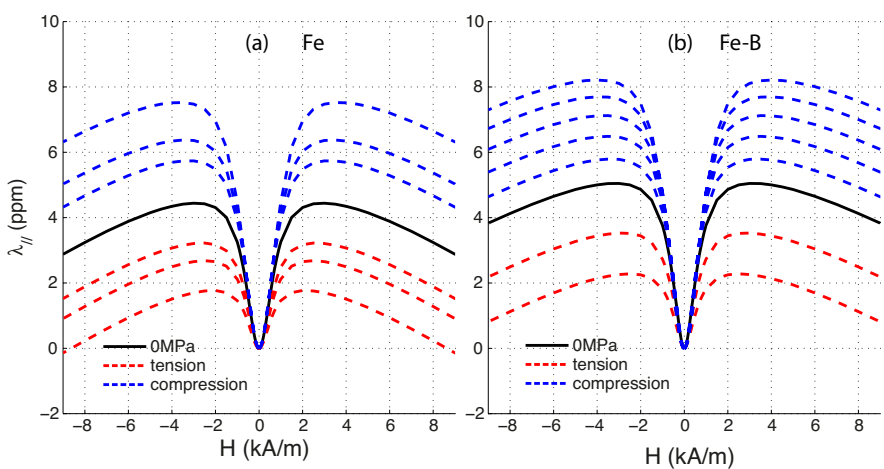

Fig. 6. Modeling - magnetostriction curves under applied stress of: (a) pure iron; (b) Fe-1.6at\%B alloy.

but are available in [4]. It has been applied to pure iron and to $\mathrm{Fe}-1.6 \mathrm{at} \% \mathrm{~B}$ alloy, using exactly the same characteristics for the pure iron $\alpha$-phase in both cases ${ }^{3}$. Figures 5, 6, 7 and 8 show the result of modeling for magnetization and magnetostriction curves under stress, magnetization $v s$ stress at different magnetic field levels (same set of values than for experiments), and piezomagnetic sensitivity $v s$ stress at the same magnetic field levels. The model reproduces accurately all behaviors for both alloys and allows a clear highlighting of the $\mathrm{Fe}_{2} \mathrm{~B}$ phase effect: decrease of magnetization, enhancement of magnetostriction; shift of the Villari reversal point; enlargement of piezomagnetic sensitivity range. Modeling of pure iron piezomagnetic sensitivity leads to lower values than those observed during experiments especially at very low field $(\leq 0.5 \mathrm{kA} / \mathrm{m})$. Measurement errors at low field of initial state of the material may be at the origin of this discrepancy, not observed for other quantities. At higher magnetic field, and thanks to the $\mathrm{Fe}_{2} \mathrm{~B}$ phase, the piezomagnetic sensitivity of pure iron becomes lower than the piezomagnetic sensitivity of $\mathrm{Fe}-1.6 \mathrm{at} \% \mathrm{~B}$ in accordance with the modeling.

\section{CONCLUSION}

It is shown in this paper that the presence of the $\mathrm{Fe}_{2} \mathrm{~B}$ phase inside the Fe matrix has first of all an average effect on

\footnotetext{
${ }^{3}$ The average loading direction is $\langle 421\rangle$ in the standard triangle, close to isotropic conditions $<431>$ [4])
}

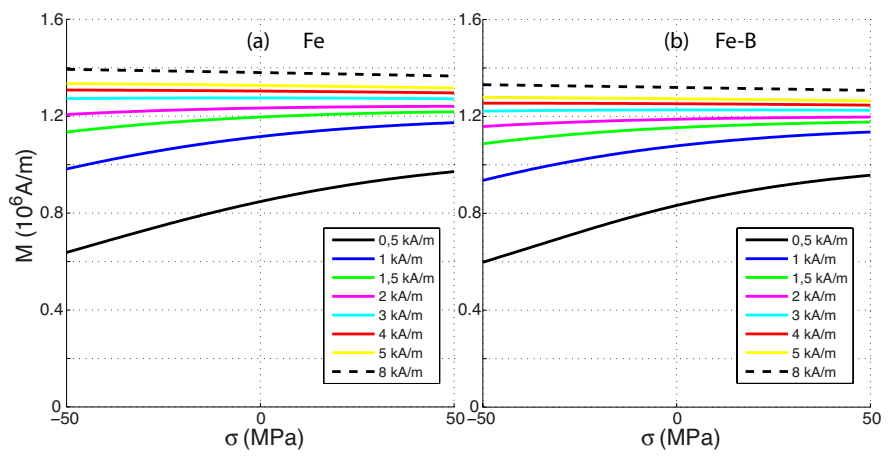

Fig. 7. Modeling - piezomagnetic curves at different magnetic field level of: (a) pure iron; (b) Fe-1.6at\%B alloy.

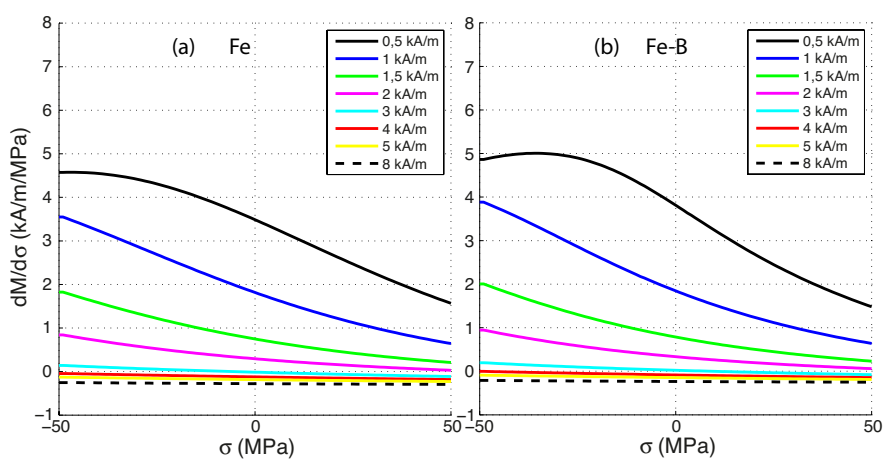

Fig. 8. Modeling - piezomagnetic sensitivity curves at different magnetic field level of: (a) pure iron; (b) Fe-1.6at\%B alloy.

magnetic, magnetostrictive and piezomagnetic quantities. On the other hand, a composite effect has been demonstrated that enhance the piezomagnetic behavior of composite comparing to the single phase material. These experimental observations have been supported by a biphasic magnetomechanical modeling whose results are in accordance with the experiments.

\section{REFERENCES}

[1] R. Grössinger, R. Turtelli, N. Mehmood, IEEE Transactions on Magnetics, 44, 11 (2008), 3001-3004.

[2] F. Moret, R. Baccino, P. Martel, L. Guetaz, J. de Physique IV, 06 (C2) (1996), 281-289.

[3] M. B. S. Dias, C. Bormio-Nunes, C. J. Pacheco, V. de Oliveira Machado and O.Hubert, Smart Mater. Struct., 24 (2015) 105004 (7pp)

[4] C. Bormio-Nunes, O. Hubert, J. of Magnetism and Magnetic Materials, 393, 11 (2015), 404-418

[5] O. Hubert, K. J. Rizzo, J. of Magnetism and Magnetic Materials, 320 (2008), e979-e982.

[6] O. Hubert, L. Daniel, R. Billardon J. of Magnetism and Magnetic Materials, 254-255, 1 (2003), 352-354.

[7] D. C. Jiles, C. C. H. Lo, Sensors and Actuators, A 106 (2003), 3-7.

[8] R.M. Bozorth, "Ferromagnetism", ed. D. Van Norstand, N.Y. 1951.

[9] A. Iga, Y. Tawara, A. Yanase, J. Phys. Soc. Japan, 21 (1966), 404

[10] W. Coene, F. Hakkens, R. Coehoorn, D. B. de Mooij, C. de Waard, J. Fidler, R. Grössinger, J. of Magnetism and Magnetic Materials, 96 , (1991), 189-196

[11] C. Bormio-Nunes, C.T. Santos, M.B.S. Dias, M. Doerr, S. Granovsky, M. Loewenhaupt, J. of Alloys and Compounds, 539 (2012), 226-232.

[12] L. Daniel, O. Hubert, N. Buiron, R. Billardon. J. of the Mechanics and Physic of Solids, 56, (2008), 1018-1042

[13] R. Hill, J. of the Mechanics and Physic of Solids, 13 (1965), 89-101. 